\title{
A nomogram to predict prognosis of patients with unresected hepatocellular carcinoma undergoing radiotherapy: a population-based study
}

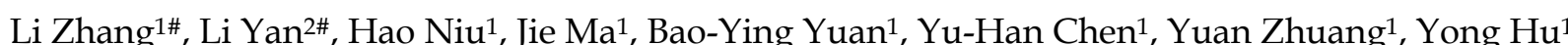
Zhao-Chong Zeng ${ }^{1 凶}$, Zuo-Lin Xiang ${ }^{3 凶}$

1. Department of Radiation Oncology, Zhongshan Hospital, Fudan University, 180 Feng Lin Road, Shanghai 200032, China

2. Department of Radiation Oncology, Eye \& ENT Hospital, Fudan University, Shanghai, 200031, China

3. Department of Radiation Oncology, Shanghai East Hospital, Tongji University School of Medicine, 150 Jimo Road, Shanghai 200120, China

\#These authors contributed equally to this work.

$\triangle$ Corresponding authors: Dr. Zhao-Chong Zeng, email: zeng.zhaochong@zs-hospital.sh.cn; and Dr. Zuo-Lin Xiang, email: xiangzuolinmd@hotmail.com

(c) The author(s). This is an open access article distributed under the terms of the Creative Commons Attribution License (https://creativecommons.org/licenses/by/4.0/). See http://ivyspring.com/terms for full terms and conditions.

Received: 2018.10.02; Accepted: 2019.05.26; Published: 2019.07.25

\begin{abstract}
Background: Radiotherapy is a primary treatment strategy for patients with unresectable hepatocellular carcinoma (HCC); however, the prognostic factors among HCC patients who have received radiotherapy but not undergone surgery have not been systematically studied. Thus, the prognostic factors were investigated in this study based on the Surveillance, Epidemiology, and End Results (SEER) Medicare database.

Methods: A screening process was used for select cases from the SEER database. Survival was analyzed using the Kaplan-Meier method and log-rank test, the Cox proportional hazards regression model, and a competing risk model. A nomogram was established for predicting 1-and 3-year overall survival (OS) of patients.

Results: A total of $1305 \mathrm{HCC}$ patients who received radiotherapy but had not undergone surgery were included in this study and divided into training $(n=1175)$ and validation cohorts $(n=130)$. Patients in the training cohort had a 1-year OS rate of $30.9 \pm 1.3 \%$, a 3-year OS rate of $10.0 \pm 1.0 \%$, and a median survival rate of 6.0 months (range, 5.4-6.6 months). Race $(p=0.025)$, T stage $(p<0.001)$, N stage $(p<0.001)$, M stage $(p<$ $0.001)$, and chemotherapy $(p<0.001)$ were identified as independent risk factors by multivariate analyses in the training cohort, while sex, age, grade, marital status, and insurance status were not independent factors. Survival in patients who received radiotherapy was worse with respect to the following characteristics: black race; higher $\mathrm{T}, \mathrm{N}$, or $\mathrm{M}$ stage; and no chemotherapy. A nomogram was established based on the results of the multivariate analysis, which was internally validated by a concordance index ( $\mathrm{C}$-index) of $0.731 \pm 0.016$ and a group of calibration plots. External validation was carried out and the C-index was $0.738 \pm 0.049$, which demonstrated the effectiveness of the nomogram we constructed.

Conclusions: Race, $T$ stage, $N$ stage, $M$ stage, and chemotherapy were independent risk factors for survival of HCC patients who received radiotherapy but had not undergone surgery. A validated nomogram was formulated to predict 1- and 3-year OS in these patients based on individual clinical characteristics.
\end{abstract}

Key words: hepatocellular carcinoma, radiotherapy, prognosis, nomogram

\section{Introduction}

As the most common pathologic type of primary liver cancer and the sixth most prevalent cancer worldwide, hepatocellular cancer (HCC) are frequent with 782,500 new cases each year and the incidence of deaths is on the rise[1,2]. Most patients with HCC are diagnosed at an intermediate-to-advanced stage[3]. Less than $20 \%$ of patients with HCC are eligible for potentially curative surgical resection or liver transplantation therapy due to multi-centric tumors, vascular invasion, extrahepatic metastases, or other co-morbidities[4]. Radiotherapy is a primary and useful strategy for such patients with unresectable HCC; however, there are few reports with a focus on prognosis among a large sample of unresectable HCC 
patients who have received radiotherapy.

A nomogram represents as intuitive graphical solution of a statistical predictive model and generates a numerical probability of a clinical event, which has been widely used in predicting survival in cancer patients[5]; however, a nomogram that predicts overall survival (OS) in HCC patients who have received radiotherapy but have not undergone surgery does not exist. To fully identify the predictive factors which influenced the prognosis of HCC patients treated by radiotherapy alone, we analyzed medical records from the Surveillance, Epidemiology, and End Results (SEER) database (http://seer. cancer.gov/) and developed a nomogram to visually predict the prognosis of these patients.

\section{Methods}

\section{Ethics statement}

The Ethics Committee of the Zhongshan Hospital, Fudan University exempted the study, because the SEER is a public database and no personal information is included.

\section{Study patients and design}

SEER (Incidence - SEER 18 Regs Custom Data with additional treatment fields, Nov 2016 Sub, 1973 2014 varying) data were obtained via the SEER ${ }^{*}$ Stat software (version 8.3.5; http://seer.cancer.gov/ seerstat/). To acquire sufficient data from the database, the selection process is shown in Figure 1. Briefly, patients with labeled primary sites C22.0-Liver were carefully reviewed. The inclusion criteria were as follows: (1) no history of other malignancies before HCC diagnosis; (2) a positive pathologic diagnosis; (3) received radiation, but had not undergone surgery; and (4) follow-up data is available. The exclusion criteria were as follows: (1) without HCC; (2) not first tumor; (3) without detailed TNM stage information; (4) performed surgery or unknown; (5) without radiotherapy or unknown; (6) not beam radiation. Finally, cases diagnosed before 2004 were excluded because there was no stage information of them. And OS was from that date of diagnosis to the date of death.

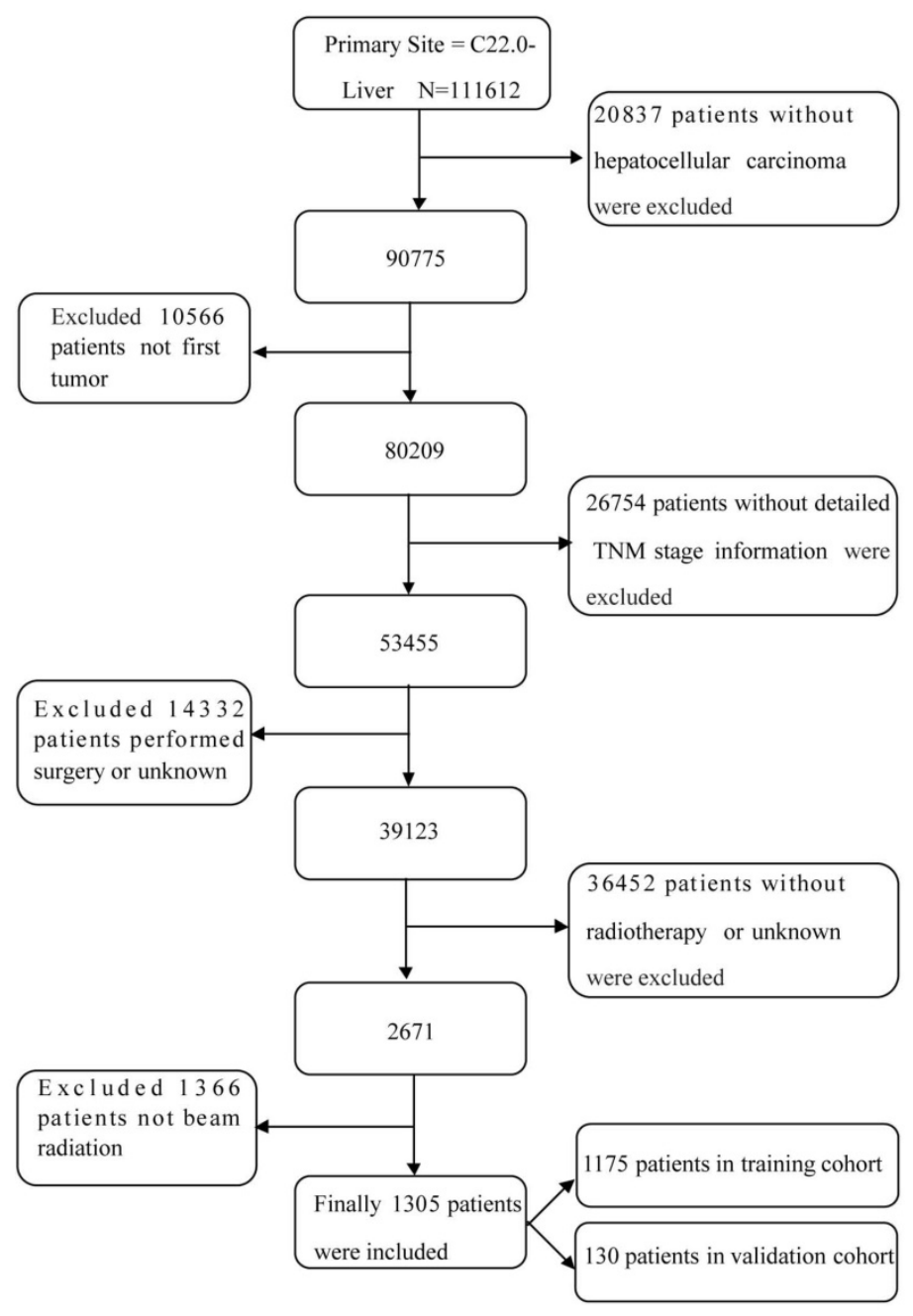

Figure 1. The flow diagram of the selection process for the study. 
For each patient, we collected relevant sociodemographic data (race, sex, and age), final pathologic analysis (histologic type and grade, and T, $\mathrm{N}$, and $\mathrm{M}$ stages), therapy (radiotherapy and chemotherapy), and follow-up data (cause of death, HCC-specific death, and survival time) from the SEER database. Ninety percent of patients were randomly divided into the training cohort and $10 \%$ of patients were classified into the validation cohort to externally validate the nomogram.

\section{Statistical analysis}

In the training cohort, survival curves for each clinicopathologic factor were depicted using the Kaplan-Meier method and compared using the log-rank test. Then, the significant variables were validated by competing risk analysis and subsequently analyzed by the Cox regression model for multivariate analysis.

A nomogram was built on the basis of the results of multivariate analysis. Bootstrap resampling was used for validation of the nomogram and calibration curve construction, as previously reported[6, 7]. The performance of the nomogram was internally measured by the concordance index (C-index) and assessed by comparing the nomogram-predicted probability with the observed probability. Then, the nomogram was further validated by comparing the nomogram-predicted probability of the patients in the validation cohort with actual survival.

Statistical analyses were carried out using SPSS 24.0 (IBM Corp., Armonk, NY, USA) and the package of ggplot, rms of R 3.4 .3 (https://www.r-project. org/). All statistical tests were 2-tailed and a $p<0.05$ was considered statistically significant.

\section{Results}

\section{Patient characteristics}

As shown in Figure 1, a total of 1305 HCC patients who had received radiotherapy but had not undergone surgery were included according to the screening criteria mentioned above between 2004 and 2014; 1175 patients were randomly divided into the training cohort and 130 patients were divided into the validation cohort. As shown in Table 1, most of the patients in the training cohort had the following characteristics: white race $(71.3 \%)$; male gender (82.9\%); 51-60 years of age (36.5\%); Gx (73.2\%); and stage IV $(65.1 \%)$. Of the patients, $44.9 \%$ had received chemotherapy, approximately $50 \%$ of the patients were married, and $75.8 \%$ of the patients were insured at the time of diagnosis.

\section{Survival analysis}

As shown in Figure 2, the patients in the training cohort had a 1 -year OS of $30.9 \pm 1.3 \%$, a 3 -year OS of $10.0 \pm 1.0 \%$, and a median survival of 6.0 months (range, 5.4-6.6 months). Univariate analyses revealed that race $(p=0.017)$, pathologic grade $(p=0.004)$, $\mathrm{T}$ stage $(p<0.001), \mathrm{N}$ stage $(p<0.001), \mathrm{M}$ stage $(p<$ $0.001)$, stage $(p<0.001)$, chemotherapy $(p<0.001)$, and insurance status $(p<0.001)$ were significant prognostic factors, as shown in Figure 3 and Supplementary Figure 1 , while sex $(p=0.260)$, age $(p=$ $0.101)$, and marital status $(p=0.475)$ were not significantly correlated with OS. The HCC-specific survival curves based on the competing risk model were summarized in Figure 4, and show consistent results compared to the univariate analysis.

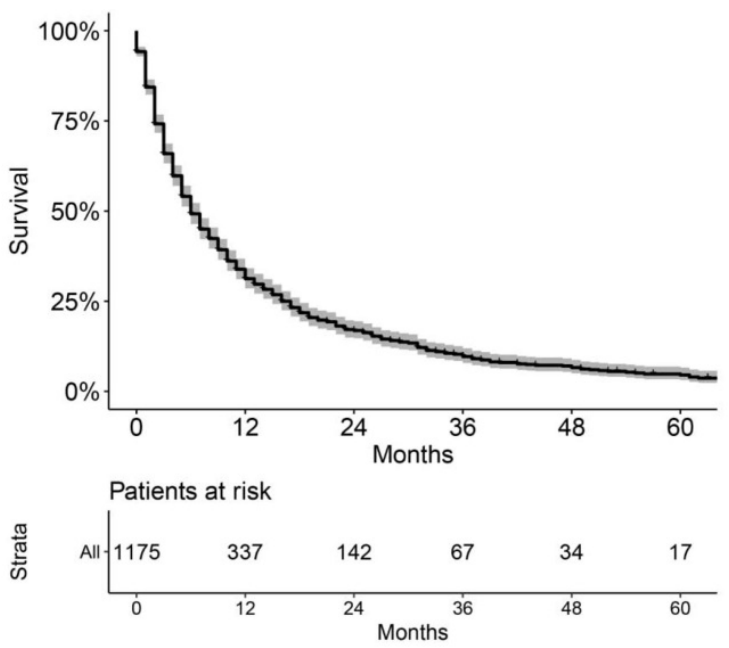

Figure 2. Overall Kaplan-Meier survival curve of the included patients in training cohort.

The significant factors were further analyzed in multivariate analysis. The results showed that race $(p$ $=0.025), \mathrm{T}$ stage $(p<0.001), \mathrm{N}$ stage $(p<0.001), \mathrm{M}$ stage $(p<0.001)$, and chemotherapy $(p<0.001)$ were independent prognosis factors, while grade $(p=0.255)$ and insurance $(p=0.634)$ were not considered as independent risk factors (Table 1). The variable stage was not included in the multivariate analysis because the variable stage was not independent from the $T, N$, and $\mathrm{M}$ stages. As the Odds Ratio(OR) values in Table 1 show, black race, higher $\mathrm{T}, \mathrm{N}$, or $\mathrm{M}$ stage, and no chemotherapy were associated with a worse survival.

\section{Construction and validation of the nomogram}

The nomogram was developed and included the following variables based on the final multivariate model, as shown in Figure 5A: race; T, N, and M stage; and chemotherapy. The calibration plots based on internal bootstrap resampling validation were illustrated in Figure 5B. The C-index for prediction of OS was $0.731 \pm 0.016$, indicating that the nomogram is in good agreement with the actual observation for 
HCC patients who had received radiotherapy but had not undergone surgery. Furthermore, the validation cohort data were used for external validation of the nomogram. After comparing the nomogrampredicted survival with the actual survival of patients
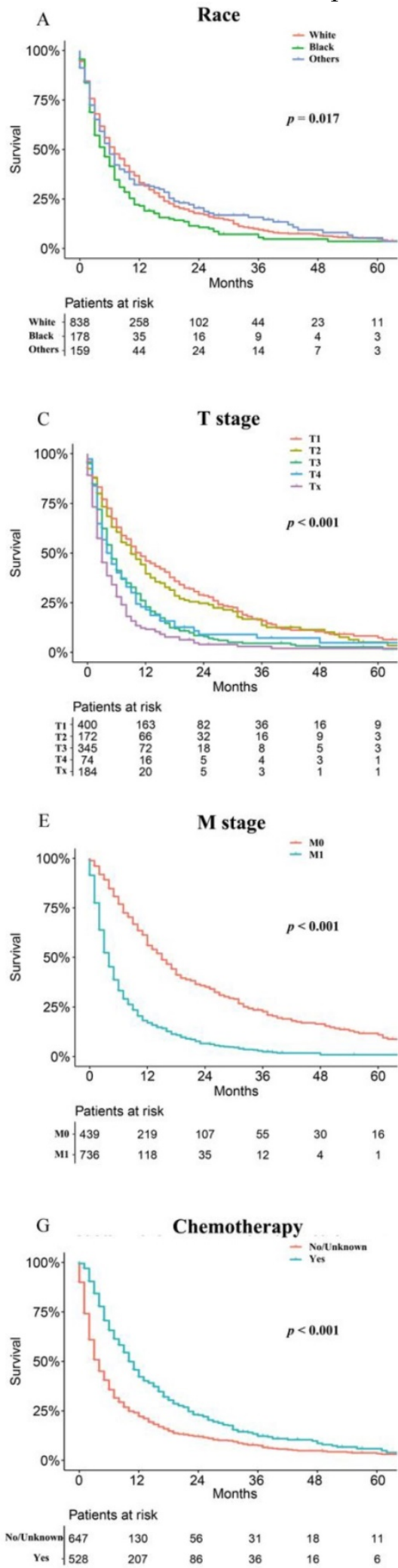

in the validation cohort, a C-index of $0.738 \pm 0.049$ was obtained based on the calibration plots shown in Figure 5C. Therefore, the nomogram reliably predicted the 1- and 3-year OS rates.
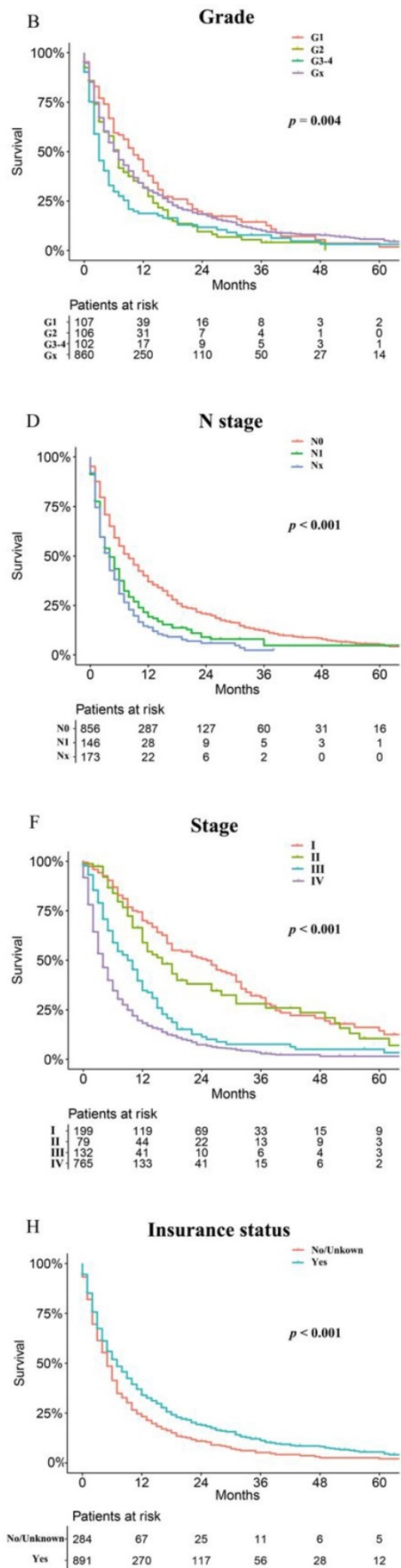

Figure 3. Overall Kaplan-Meier survival curves for patients in training cohort according to (A) Race, (B) Grade, (C) T stage, (D) N stage, (E) M stage, (F) Stage, (G) Chemotherapy, (H) Insurance status. 

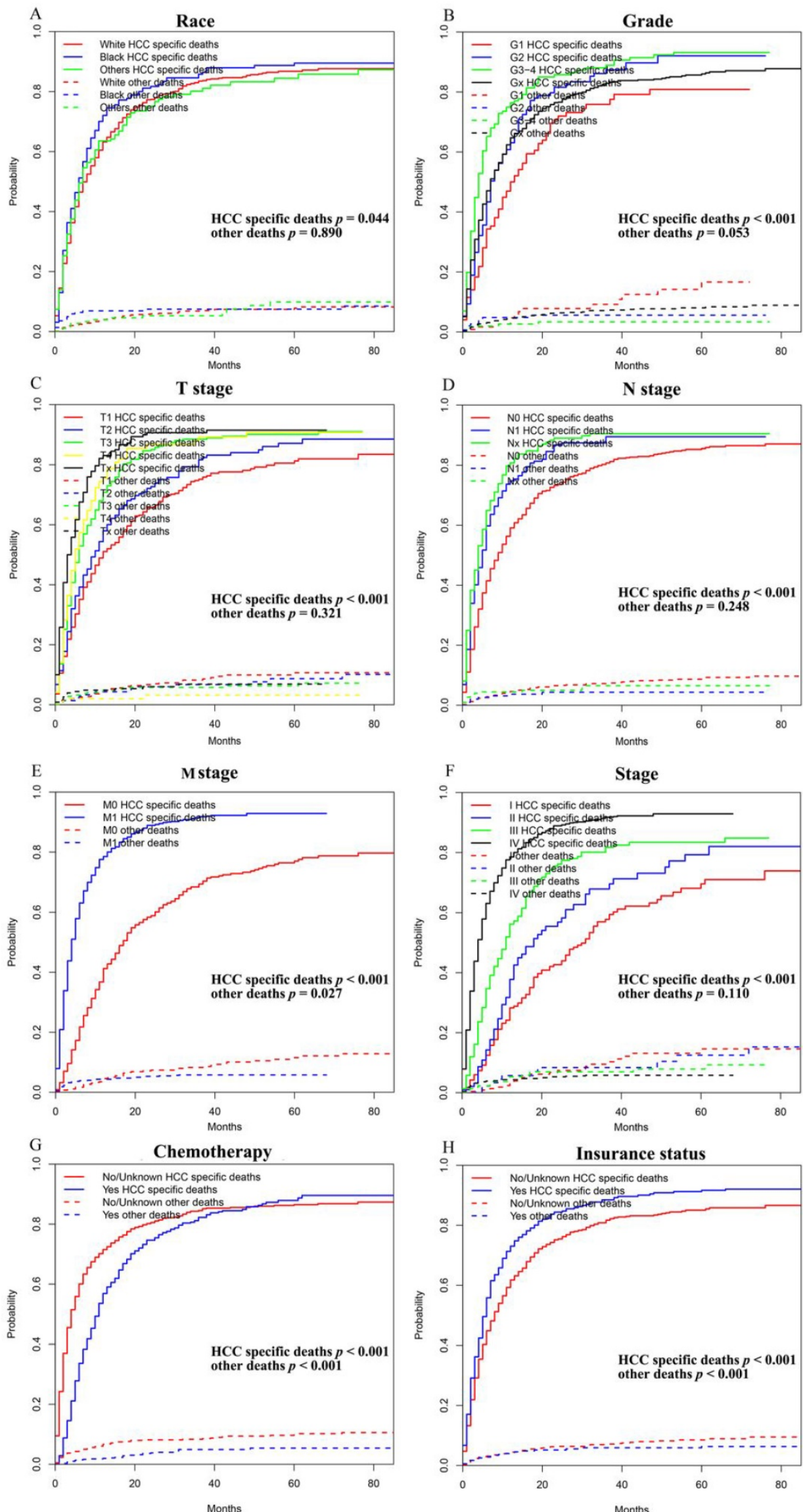

Figure 4. Competing risk analyses for patients in training cohort according to (A) Race, (B) Grade, (C) T stage, (D) N stage, (E) M stage, (F) Stage, (G) Chemotherapy, (H) Insurance status. 
A

Points

Race

\section{White}

Tstage

Others Black

Nstage

$\begin{array}{llll}\text { T1 } & & \text { T3 } & \text { Tx } \\ & \text { Nx } & & \text { T4 } \\ & & & \end{array}$

Mstage

No N1

Chemotherapy

M0

M1

Total Points

Yes

No/Unknown

1-year survival

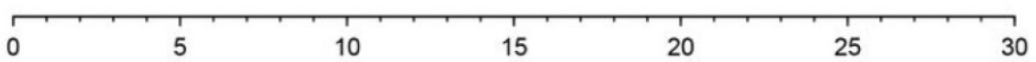

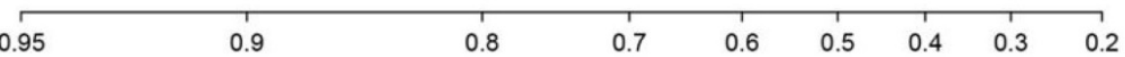

3-year survival

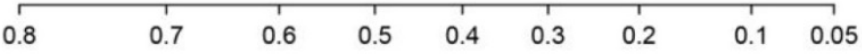

\section{B}
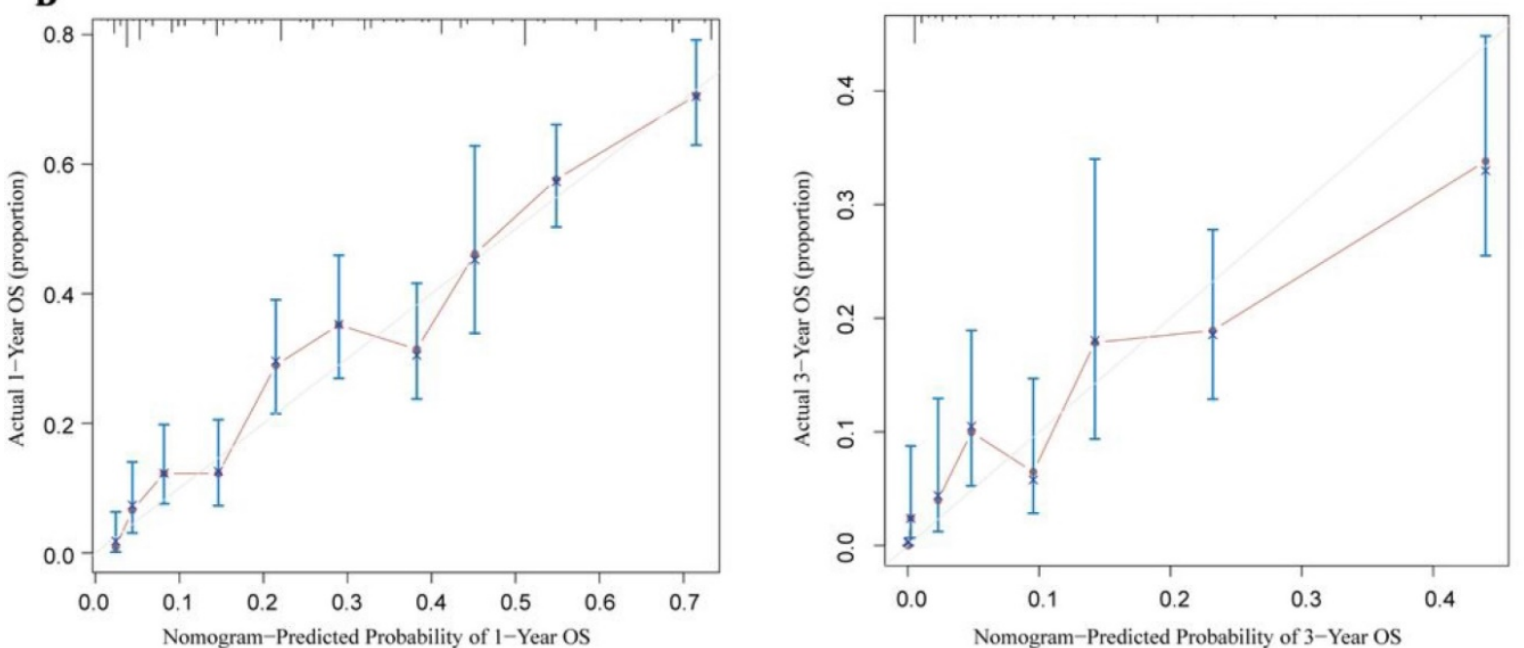

C
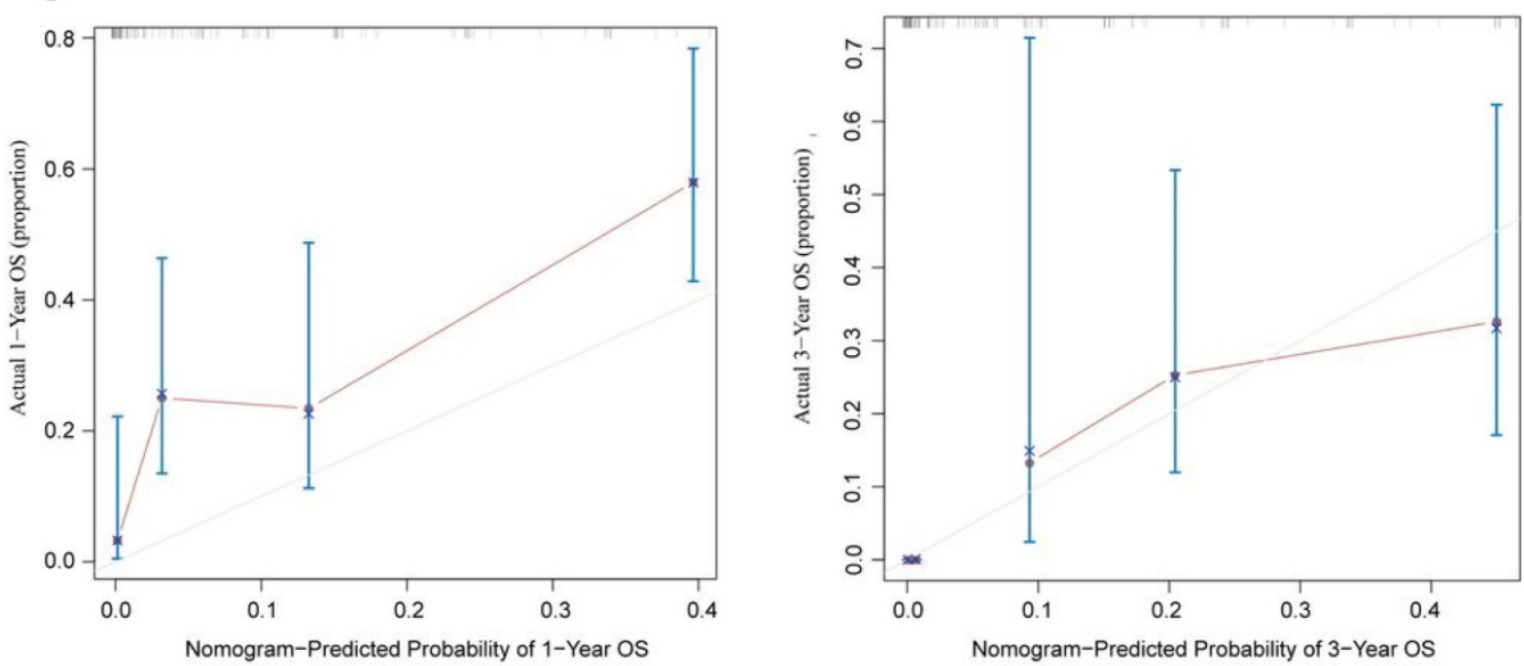

Figure 5. A nomogram for prediction of 1- and 3-year OS rates of patients with unresected $\mathrm{HCC}$ undergoing radiotherapy (A); Calibration curve of the nomogram predicting 1-year and 3-year OS rates of unresected HCC patients undergoing radiotherapy in training cohort (B); Calibration curve of the nomogram predicting 1-year and 3-year OS rates of patients with unresected $\mathrm{HCC}$ undergoing radiotherapy in the validation cohort (C). 
Table 1. Patient characteristics and 1-, and 3-year OS rates

\begin{tabular}{|c|c|c|c|c|c|c|c|}
\hline Variable & Number of patients & OR(95\%CI) & 1-year OS (\%) & 3-year OS(\%) & Median survival & $\begin{array}{l}p \text { value } \\
\text { (univariate analysis) }\end{array}$ & $\begin{array}{l}p \text { value } \\
\text { (multivariate analysis) }\end{array}$ \\
\hline Total cases & $1175(100 \%)$ & & $0.309 \pm 0.013$ & $0.100 \pm 0.010$ & $6.0(5.4-6.6)$ & & \\
\hline Race & & & & & & 0.017 & 0.025 \\
\hline White & $838(71.3 \%)$ & Reference & $0.332 \pm 0.017$ & $0.094 \pm 0.012$ & $7.0(6.0-8.0)$ & & \\
\hline Black & $178(15.1 \%)$ & $1.287(1.083-1.530)$ & $0.215 \pm 0.032$ & $0.055 \pm 0.019$ & $5.0(3.6-6.4)$ & & \\
\hline Other & $159(13.5 \%)$ & $0.982(0.814-1.184)$ & $0.322 \pm 0.039$ & $0.157 \pm 0.032$ & $6.0(5.4-6.6)$ & & \\
\hline Sex & & & & & & 0.260 & not included \\
\hline Male & $974(82.9 \%)$ & Reference & $0.307 \pm 0.016$ & $0.091 \pm 0.011$ & $6.0(5.4-6.6)$ & & \\
\hline Female & $201(17.1 \%)$ & $0.908(0.769-1.074)$ & $0.335 \pm 0.039$ & $0.124 \pm 0.027$ & $7.0(5.2-8.8)$ & & \\
\hline Age(years) & & & & & & 0.101 & not included \\
\hline $0-50$ & $112(9.5 \%)$ & Reference & $0.267 \pm 0.044$ & $0.112 \pm 0.034$ & $5.0(3.8-6.2)$ & & \\
\hline $51-60$ & $429(36.5 \%)$ & $1.132(0.899-1.425)$ & $0.262 \pm 0.022$ & $0.068 \pm 0.016$ & $6.0(5.2-6.8)$ & & \\
\hline $61-70$ & $336(28.6 \%)$ & $0.907(0.715-1.151)$ & $0.352 \pm 0.028$ & $0.143 \pm 0.023$ & $8.0(5.9-10.1)$ & & \\
\hline $71-80$ & $221(18.8 \%)$ & $1.007(0.785-1.292)$ & $0.358 \pm 0.033$ & $0.078 \pm 0.020$ & $7.0(5.5-8.5)$ & & \\
\hline $81+$ & $77(6.6 \%)$ & $1.060(0.770-1.460)$ & $0.354 \pm 0.058$ & $0.078 \pm 0.039$ & 7.0(4.6-9.4) & & \\
\hline Pathologic grade & & & & & & 0.004 & 0.255 \\
\hline G1 & $107(9.1 \%)$ & Reference & $0.401 \pm 0.051$ & $0.144 \pm 0.039$ & $10.0(7.5-12.5)$ & & \\
\hline G2 & $106(9 \%)$ & $1.333(0.993-1.789)$ & $0.319 \pm 0.047$ & $0.041 \pm 0.022$ & $7.0(5.8-8.2)$ & & \\
\hline G3-4 & $102(8.7 \%)$ & $1.644(1.224-2.208)$ & $0.187 \pm 0.040$ & $0.078 \pm 0.029$ & $3.0(1.9-4.1)$ & & \\
\hline Gx & $860(73.2 \%)$ & $1.140(0.911-1.426)$ & $0.315 \pm 0.017$ & $0.100 \pm 0.012$ & $7.0(6.2-7.8)$ & & \\
\hline T stage & & & & & & $<0.001$ & $<0.001$ \\
\hline $\mathrm{T} 1$ & $400(34.0 \%)$ & Reference & $0.460 \pm 0.026$ & $0.159 \pm 0.022$ & $11.0(8.9-13.1)$ & & \\
\hline $\mathrm{T} 2$ & $172(14.6 \%)$ & $1.132(0.926-1.383)$ & $0.396 \pm 0.039$ & $0.136 \pm 0.031$ & $9.0(6.2-11.8)$ & & \\
\hline T3 & $345(29.4 \%)$ & $1.765(1.500-2.078)$ & $0.228 \pm 0.024$ & $0.045 \pm 0.014$ & $5.0(4.2-5.8)$ & & \\
\hline $\mathrm{T} 4$ & $74(6.3 \%)$ & $1.708(1.310-2.228)$ & $0.215 \pm 0.049$ & $0.072 \pm 0.033$ & $4.0(2.1-5.9)$ & & \\
\hline $\mathrm{Tx}$ & $184(15.7 \%)$ & $2.482(2.050-3.005)$ & $0.116 \pm 0.024$ & $0.029 \pm 0.015$ & $3.0(2.2-3.8)$ & & \\
\hline N stage & & & & & & $<0.001$ & $<0.001$ \\
\hline No & $856(72.9 \%)$ & Reference & $0.370 \pm 0.017$ & $0.120 \pm 0.013$ & $8.0(6.9-9.1)$ & & \\
\hline N1 & $146(12.4 \%)$ & $1.518(1.255-1.837)$ & $0.192 \pm 0.034$ & $0.048 \pm 0.023$ & $4.0(2.5-5.5)$ & & \\
\hline $\mathrm{Nx}$ & $173(14.7 \%)$ & $1.851(1.554-2.203)$ & $0.139 \pm 0.027$ & $0.024 \pm 0.015$ & $4.0(3.1-4.9)$ & & \\
\hline M stage & & & & & & $<0.001$ & $<0.001$ \\
\hline M0 & $439(37.4 \%)$ & Reference & $0.560 \pm 0.025$ & $0.226 \pm 0.024$ & $15.0(12.8-17.2)$ & & \\
\hline M1 & $736(62.6 \%)$ & $2.820(2.447-3.250)$ & $0.171 \pm 0.014$ & $0.024 \pm 0.007$ & $4.0(3.6-4.4)$ & & \\
\hline Stage & & & & & & $<0.001$ & not included \\
\hline I & $199(16.9 \%)$ & Reference & $0.700 \pm 0.035$ & $0.311 \pm 0.041$ & $25.0(18.8-31.2)$ & & \\
\hline II & $79(6.7 \%)$ & $1.244(0.904-1.711)$ & $0.589 \pm 0.059$ & $0.281 \pm 0.059$ & $16.0(11.5-20.5)$ & & \\
\hline III & $132(11.2 \%)$ & $2.253(1.728-2.936)$ & $0.348 \pm 0.045$ & $0.076 \pm 0.028$ & $\mathrm{v}$ & & \\
\hline IV & $765(65.1 \%)$ & $3.628(2.968-4.434)$ & $0.183 \pm 0.015$ & $0.029 \pm 0.007$ & $4.0(3.6-4.4)$ & & \\
\hline Chemotherapy & & & & & & $<0.001$ & $<0.001$ \\
\hline No/Unkown & $647(55.1 \%)$ & Reference & $0.222 \pm 0.017$ & $0.075 \pm 0.012$ & $4.0(3.4-4.6)$ & & \\
\hline Yes & $528(44.9 \%)$ & $0.566(0.498-0.643)$ & $0.422 \pm 0.023$ & $0.122 \pm 0.017$ & $10.0(8.9-11.1)$ & & \\
\hline Marital status & & & & & & 0.475 & not included \\
\hline Married & $607(51.7 \%)$ & Reference & $0.313 \pm 0.020$ & $0.083 \pm 0.014$ & $6.0(5.2-6.8)$ & & \\
\hline Others & $568(48.3 \%)$ & $1.047(0.923-1.187)$ & $0.312 \pm 0.020$ & $0.109 \pm 0.015$ & $7.0(6.0-8.0)$ & & \\
\hline Insurance status & & & & & & $<0.001$ & 0.634 \\
\hline No/Unkown & $284(24.2 \%)$ & Reference & $0.232 \pm 0.025$ & $0.052 \pm 0.015$ & $5.0(4.2-5.8)$ & & \\
\hline Yes & $891(75.8 \%)$ & $0.759(0.659-0.874)$ & $0.340 \pm 0.017$ & $0.113 \pm 0.013$ & $7.0(6.0-8.0)$ & & \\
\hline
\end{tabular}

\section{Discussion}

In the current study we analyzed the risk factors for HCC patients who received radiotherapy but had not undergone surgery. Greater than 1000 patients were analyzed and we showed that race, $\mathrm{T}, \mathrm{N}$, and $\mathrm{M}$ stages, and chemotherapy were independent prognostic factors and established a nomogram to visually and vividly predict patient survival. A small portion of the patients were isolated for external validation to demonstrate the reliability of the nomogram. Finally, both internal and external validations demonstrated the accuracy and effectiveness of the nomogram. The nomogram, a statistical model based on a combination of significant factors, will contribute to evaluating the individualized prognosis and instructing treatment of the patients. In this way, treatment guidelines and prediction of outcomes would be more efficient and accurate for doctors and patients. With its wide range of clinical applications, the nomogram is a very reliable and available predictive system.

Most unresectable HCCs have invaded the intrahepatic large vessels or metastasized and are associated with extremely poor outcomes[8]. Approximately $30 \%$ of patients were T3 and $60 \%$ were M1 in the current study, with a 1-year OS rate of $30 \%$. In a multicenter phase I trial, $56 \%$ of unresectable 
patients who received radiotherapy had a partial response and $28 \%$ showed stable disease, with a 1 -year OS rate of $61 \%$ [9]. Therefore, radiotherapy is a promising, non-invasive therapeutic modality for patients with unresectable HCC. Radiotherapy may also potentially serve as a bridging therapy for patients awaiting transplantation and can be used in combination with other locoregional or systemic therapies, such as transarterial chemoembolization and sorafenib[10-13].

Blacks had a poorer prognosis in our analysis and several other reports[14,15], likely due to the higher rates of hepatitis $C$ virus and hepatitis $B$ virus infections. Sex and age were not prognostic factors in the current study, which is consistent with a previous report[16], though younger patients and female patients usually have better survival in most types of cancer.

A number of studies have indicated that pathologic grade is a predictor of survival in patients with HCC $[17,18]$. In the present study, pathologic grade was strongly associated with 1- and 3-year survival, but pathologic grade was not an independent prognostic factor. Poorly differentiated lesions are significantly larger in size and cannot be resected[19]. Additionally, TNM stage is known to significantly predict survival in patients with unresectable HCCs, which was also demonstrated in our study. Tumor size $>5 \mathrm{~cm}$ in size represents a poor prognosis for HCC and is a predictive factor for early HCC recurrence[20, 21]. Additionally, large tumors have a higher risk of vascular invasion and higher tumor grade[22]. HCCs with and without lymph node metastases have different survival rates (5 months vs. 12 months, respectively)[23]. Metastasis is a leading cause of early recurrence and poor prognosis in HCC patients. Patients with metastatic HCC often do not survive $>1$ year[24].

Approximately $45 \%$ of patients in our study were confirmed to have received chemotherapy concurrent with radiotherapy, and these patients had a significantly better survival. In recent years, a variety of novel chemotherapeutic agents have become available to improve patients' survival. The multi-kinase inhibitor, sorafenib, is a standard therapy for advanced HCC and is often combined with chemotherapy to increase safety and efficiency[25, 26]. The expression of PD-L1 has been reported to be correlated with poor survival of HCC patients who have received radiotherapy[27]. A study in a murine model showed that the combination of anti-PD-L1 and radiation significantly improved the anti-tumor effect, suggesting a novel combination strategy of immunoradiotherapy in HCC[28].
Several previous studies have revealed HCC-specific prognostic variables. For instance, the serum level of AFP was most commonly used biomarker in HCC diagnosis and prognosis[29]. However, AFP expression in many cases of liver cancer is not elevated[30]. And the patient gender effect have potentially influenced AFP levels, which increased in women[31]. And overexpression of PARPBP correlated with tumor progression and poor prognosis in HCC patients after surgery[32]. Although such variables may be potentially useful for the prognosis prediction, the reported prognostic factors for HCC OS are different among studies[33]. Compared with the prognostic variables, nomograms can provide more personalized assessment for the prognosis of patients. Wang et al. developed a nomogram that combined BMI, tumor stage, distant metastases, HBsAg, LDH, GGT and ALB, for 3- and 5-year OS in patients with AFP-negative HCC in single institution[34]. Several published nomograms have been established for predicting prognosis after radiotherapy for brain metastases and lymph node metastasis(LNM) from HCC. Park Y et al. developed a nomogram predicting the survival of 97 patients with brain metastasis from HCC treated with whole brain radiotherapy (a C-index of 0.74 ). This retrospective study was conducted in a relatively small study population due to the low incidence of brain metastasis from HCC[33]. Kim Y et al. developed a nomogram to predict 2-year OS with good accuracy (a C-index of 0.72) in patients who received radiotherapy for abdominal LNM from HCC. This nomogram was based on a relatively large sample size with 228 patients through the use of a multi-institutional study. The nomogram combined the significant prognostic factors, including Child-Pugh classification, status of intrahepatic tumor, presence of distant metastasis, location and number of metastatic abdominal lymph nodes(LNs), serum level of AFP, and the $\mathrm{LN}$ response to radiotherapy[35]. WEE et al. Developed a nomogram for predicting 6-month survival with a C-index of 0.77 in HCC patients treated with radiotherapy for LNM. In consideration of the inclusion of both intra-abdominal and extra-abdominal LN metastasis, the LN-related symptoms are highly associated with the patients' survival, which may affect the accuracy of the prognostic model[36]. Based on the results of the present study, a nomogram was formulated using race, $\mathrm{T}, \mathrm{N}$, and $\mathrm{M}$ stages, and chemotherapy. We constructed the nomogram for predicting 1- and 3-OS based on the clinical information in both internal validation with C-index of $0.731 \pm 0.016$ and external validation with $\mathrm{C}$-index of $0.738 \pm 0.049$ in unresected HCC patients undergoing radiotherapy. This is the 
first study that developed a nomogram for unresected HCC patients undergoing radiotherapy. Though a large number of samples, tumor markers have not been included in the nomogram.

There were several limitations in this study that should be noted. First, because the SEER database did not provide detailed information regarding radiotherapy and chemotherapy, we could not calculate the effect of radiation technology, dose, and interval, and chemotherapeutic reagents. Second, the reasons that patients did not undergo surgery were not always recorded. Some patients did not undergo surgery for reasons other than the tumor, such as hepatitis or poor liver function. Third, the patients were all from the USA, thus the results might not be applicable to other populations.

In conclusion, we showed that race, $\mathrm{T}, \mathrm{N}$, and $\mathrm{M}$ stage, and chemotherapy are independent risk factors for survival of HCC patients who have received radiotherapy, but have not undergone surgery. We established a nomogram to facilitate visual prediction of 1- and 3-year OS based on individual clinical characteristics. Though both internal and external validation demonstrated the reliability of the nomogram, further studies are warranted.

\section{Abbreviations}

HCC, hepatocellular carcinoma; SEER, Surveillance, Epidemiology, and End Results; OS, overall survival; C-index, concordance index; OR, Odds Ratio.

\section{Supplementary Material}

Supplementary figure 1.

http://www.jcancer.org/v10p4564s1.pdf

\section{Acknowledgements}

We thank the International Science Editing Co. for editing the language.

\section{Funding}

This work was supported by the National Natural Science Foundation of China(Grant No. U1505229, 81703023) and the Natural Science Foundation of Shanghai (Grant No.17ZR1405300), Science and Technology supporting project of Shanghai (Grant No.17411962600), Pudong New Area Science and Technology Development Fund (Grant no.PKJ2018-Y02) and Shanghai municipal human resources and social security bureau (Grant No.Q2016-019).

\section{Authors' contributions}

Zhao-Chong Zeng, and Zuo-Lin Xiang conceived the study design. Data acquisition was carried out by
Li Zhang, Jie Ma, Bao-Ying Yuan, Yu-Han Chen, Yuan Zhuang, and Yong Hu. Li Zhang, Li Yan and Hao Niu did the data analysis. Li Zhang and Li Yan drafted the manuscript. Revision of the manuscript was done by Hao Niu, Zhao-Chong Zeng and Zuo-Lin Xiang. All authors contributed toward data analysis, drafting and critically revising the paper and agree to be accountable for all aspects of the work.

\section{Availability of data and material}

The datasets used and/or analysed during the current study are available from the corresponding author on reasonable request.

\section{Competing Interests}

The authors have declared that no competing interest exists.

\section{References}

1. Torre LA, Bray F, Siegel RL, et al. Global cancer statistics, 2012. CA Cancer J Clin. 2015;65(2):87-108

2. Rapisarda V, Loreto C, Malaguarnera M, et al. Hepatocellular carcinoma and the risk of occupational exposure. World J Hepatol. 2016;8(13):573-90.

3. Bruix J, Sherman M. Management of hepatocellular carcinoma. Hepatology. 2005;42(5):1208-36.

4. Guan YS, Liu Y. Interventional treatments for hepatocellular carcinoma. Hepatobiliary Pancreat Dis Int. 2006;5(4):495-500.

5. Iasonos A, Schrag D, Raj GV, et al. How to build and interpret a nomogram for cancer prognosis. J Clin Oncol. 2008;26(8):1364-70.

6. Zhan $C$, Yang $X$, Song $X$, et al. Radiotherapy vs surgery for T1-2N0M0 laryngeal squamous cell carcinoma: A population-based and propensity score matching study. Cancer Med. 2018.

7. Sun F, Ma K, Yang X, et al. A nomogram to predict prognosis after surgery in early stage non-small cell lung cancer in elderly patients. Int J Surg. 2017;42:11-6.

8. Tanaka Y, Nakazawa T, Komori S, et al. Radiotherapy for patients with unresectable advanced hepatocellular carcinoma with invasion to intrahepatic large vessels: efficacy and outcomes. J Gastroenterol Hepatol. 2014;29(2):352-7.

9. Herrmann E, Naehrig D, Sassowsky M, et al. External beam radiotherapy for unresectable hepatocellular carcinoma, an international multicenter phase I trial, SAKK 77/07 and SASL 26. Radiat Oncol. 2017;12(1):12.

10. Abdel-Rahman O, Elsayed Z. External beam radiotherapy for unresectable hepatocellular carcinoma. Cochrane Database Syst Rev. 2017;3:D11314.

11. Klein J, Dawson LA. Hepatocellular carcinoma radiation therapy: review of evidence and future opportunities. Int J Radiat Oncol Biol Phys. 2013;87(1):22-32.

12. Yoon SM, Ryoo BY, Lee SI, et al. Efficacy and Safety of Transarterial Chemoembolization Plus External Beam Radiotherapy vs Sorafenib in Hepatocellular Carcinoma With Macroscopic Vascular Invasion: A Randomized Clinical Trial. JAMA Oncol. 2018;4(5):661-9.

13. Huo YR, Eslick GD. Transcatheter Arterial Chemoembolization Plus Radiotherapy Compared With Chemoembolization Alone for Hepatocellular Carcinoma: A Systematic Review and Meta-analysis. JAMA Oncol. 2015;1(6):756-65

14. Venepalli NK, Modayil MV, Berg SA, et al. Features of hepatocellular carcinoma in Hispanics differ from African Americans and non-Hispanic Whites. World J Hepatol. 2017;9(7):391-400.

15. Stewart SL, Kwong SL, Bowlus CL, et al. Racial/ethnic disparities in hepatocellular carcinoma treatment and survival in California, 1988-2012. World J Gastroenterol. 2016;22(38):8584-95.

16. Kim M, Kay CS, Jang WI, et al. Prognostic value of tumor volume and radiation dose in moderate-sized hepatocellular carcinoma: A multicenter analysis in Korea (KROG 14-17). Medicine (Baltimore). 2017;96(24):e7202.

17. Kim SU, Jung KS, Lee S, et al. Histological subclassification of cirrhosis can predict recurrence after curative resection of hepatocellular carcinoma. Liver Int. 2014;34(7):1008-17.

18. Zhou L, Rui JA, Zhou WX, et al. Edmondson-Steiner grade: A crucial predictor of recurrence and survival in hepatocellular carcinoma without microvascular invasio. Pathol Res Pract. 2017;213(7):824-30.

19. Zimmerman MA, Trotter JF, Wachs M, et al. Predictors of long-term outcome following liver transplantation for hepatocellular carcinoma: a single-center experience. Transpl Int. 2007;20(9):747-53.

20. Lencioni R, Cioni D, Crocetti L, et al. Early-stage hepatocellular carcinoma in patients with cirrhosis: long-term results of percutaneous image-guided radiofrequency ablation. Radiology. 2005;234(3):961-7. 
21. Kuo HT, Que J, Lin LC, et al. Impact of tumor size on outcome after stereotactic body radiation therapy for inoperable hepatocellular carcinoma. Medicine (Baltimore). 2017;96(50):e9249.

22. Kee KM, Wang JH, Lin CY, et al. Validation of the 7th edition TNM staging system for hepatocellular carcinoma: an analysis of 8,828 patients in a single medical center. Dig Dis Sci. 2013;58(9):2721-8.

23. Xia $\mathrm{F}, \mathrm{Wu} \mathrm{L}$, Lau WY, et al. Positive lymph node metastasis has a marked impact on the long-term survival of patients with hepatocellular carcinoma with extrahepatic metastasis. Plos One. 2014;9(4):e95889.

24. Llovet JM, Hernandez-Gea V. Hepatocellular carcinoma: reasons for phase III failure and novel perspectives on trial design. Clin Cancer Res. 2014;20(8):2072-9.

25. Cha J, Seong J, Lee IJ, et al. Feasibility of sorafenib combined with local radiotherapy in advanced hepatocellular carcinoma. Yonsei Med J. 2013;54(5):1178-85

26. Wada Y, Takami Y, Matsushima H, et al. The Safety and Efficacy of Combination Therapy of Sorafenib and Radiotherapy for Advanced Hepatocellular Carcinoma: A Retrospective Study. Intern Med. 2018;57(10):1345-53.

27. Kim HJ, Park S, Kim KJ, et al. Clinical significance of soluble programmed cell death ligand-1 (sPD-L1) in hepatocellular carcinoma patients treated with radiotherapy. Radiother Oncol. 2018;129(1):130-5.

28. Kim KJ, Kim JH, Lee SJ, et al. Radiation improves antitumor effect of immune checkpoint inhibitor in murine hepatocellular carcinoma model. Oncotarget. 2017;8(25):41242-55.

29. A new prognostic system for hepatocellular carcinoma: a retrospective study of 435 patients: the Cancer of the Liver Italian Program (CLIP) investigators. Hepatology. 1998;28(3):751-5.

30. Asrih M, Lenglet S, Mach F, et al. Alpha-fetoprotein: a controversial prognostic biomarker for small hepatocellular carcinoma. World J Gastroenterol. 2013;19(3):328-30.

31. Li T, Qin LX, Gong X, et al. Hepatitis B virus surface antigen-negative and hepatitis $C$ virus antibody-negative hepatocellular carcinoma: clinical characteristics, outcome, and risk factors for early and late intrahepatic recurrence after resection. Cancer. 2013;119(1):126-35.

32. Yu B, Ding Y, Liao X, et al. Overexpression of PARPBP Correlates with Tumor Progression and Poor Prognosis in Hepatocellular Carcinoma. Dig Dis Sci. 2019.

33. Park Y, Kim KS, Kim K, et al. Nomogram prediction of survival in patients with brain metastases from hepatocellular carcinoma treated with whole-brain radiotherapy: a multicenter retrospective study. J Neurooncol. 2015;125(2):377-83.

34. Wang X, Mao M, He Z, et al. Development and Validation of a Prognostic Nomogram in AFP-negative hepatocellular carcinoma. Int J Biol Sci. 2019;15(1):221-8

35. Kim Y, Park HC, Yoon SM, et al. Prognostic group stratification and nomogram for predicting overall survival in patients who received radiotherapy for abdominal lymph node metastasis from hepatocellular carcinoma: a multi-institutional retrospective study (KROG 15-02). Oncotarget. 2017;8(55):94450-61.

36. Wee CW, Kim K, Chie EK, et al. Prognostic stratification and nomogram for survival prediction in hepatocellular carcinoma patients treated with radiotherapy for lymph node metastasis. Br J Radiol. 2016;89(1065):20160383. 